Review Article

\title{
Conducting Polymer-Based Composite Materials for Therapeutic Implantations: From Advanced Drug Delivery System to Minimally Invasive Electronics
}

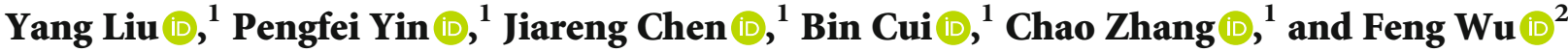 \\ ${ }^{1}$ Department of Biomedical Engineering, Sun Yat-sen University, Guangzhou, China 510006 \\ ${ }^{2}$ Bioproducts Discovery \& Development Center, University of Guelph, Ontario, Canada
}

Correspondence should be addressed to Yang Liu; liuyang56@mail.sysu.edu.cn and Feng Wu; fengwu@uoguelph.ca

Received 20 September 2019; Revised 8 December 2019; Accepted 24 December 2019; Published 6 February 2020

Academic Editor: Victor H. Perez

Copyright (๑) 2020 Yang Liu et al. This is an open access article distributed under the Creative Commons Attribution License, which permits unrestricted use, distribution, and reproduction in any medium, provided the original work is properly cited.

\begin{abstract}
Conducting polymer-based composites have recently becoming popular in both academic research and industrial practices due to their high conductivity, ease of process, and tunable electrical properties. The multifunctional conducting polymer-based composites demonstrated great application potential for in vivo therapeutics and implantable electronics, including drug delivery, neural interfacing, and minimally invasive electronics. In this review article, the state-of-the-art conducting polymerbased composites in the mentioned biological fields are discussed and summarized. The recent progress on the synthesis, structure, properties, and application of the conducting polymer-based composites is presented, aimed at revealing the structure-property relationship and the corresponding functional applications of the conducting polymer-based composites. Furthermore, key issues and challenges regarding the implantation performance of these composites are highlighted in this paper.
\end{abstract}

\section{Introduction}

Conducting polymers (CPs) can be referred to the composite materials comprising polymeric components and conductive components. The polymeric components and the conductive components of the CPs can be arranged in different structures and configurations, e.g., mixing, coating, and hierarchi$\mathrm{cal}$, to gain the designed functionality. Different from the CPs, the intrinsically conducting polymers (ICPs) can transport the electrons and holes through their unique alternating single-and-double-bond structure in the main chains, and the addition of another conductive phase is not necessary. The discovery of the ICPs dates back to the 1850s with the introduction of polyaniline [1] by Henry Letheby, but only in the 1970s did polyaniline (PANI) and other conducting polymers such as polypyrrole (PPy), polythiophene, and polyacetylene receive intense attention from the scientific and industrial communities due to the breakthrough work done by Heeger, MacDiarmid, and Shirakawa [2]. Conducting polymers are organic polymers that can conduct electricity, which are different from the conventional polymers such as polyethylene and natural rubber, and due to their unusual electrochemical and optical properties, the ICPs have greatly benefitted our society in applications such as energy storage [3], supercapacitors [4, 5], field emission [6], biosensors [7], gas sensors [8], and tissue engineering [9]. Chemical synthesis and electrochemical synthesis are generally two main methods to synthesize ICPs; in particular, electrochemistry has played a key role in the synthesis of ICPs for its finetuning in the polymer structures, compositions, and electrochemical properties. Several articles and reviews have been published on the synthesis, properties, and applications of ICPs $[10,11]$. However, to achieve a wide range of applicability, excellent mechanical properties, easy processability, and high-performance electrical, sensing, and energy-storage capabilities are required for the next-generation conducting polymer-based composites.

Compared to the bulk polymers, the conducting polymerbased composites which are formed by blending or mixing ICPs with other materials have drawn more attention, and the properties of each individual component can be efficiently integrated to achieve multifunctionality [12]. Various 
strategies have been developed to synthesize the conducting polymer-based composites, such as electrosynthesis in the presence of an insulating polymer [8], encapsulation of fibers [13], incorporation of other secondary nanoparticles to form conducting polymer nanocomposites [14], electropolymerization of different monomers (e.g., aniline and 3,4-ethylenedioxythiophene) [15], and the utilization of dopants with multiple functions (e.g., disulfide biotin) [16], and the in situ integration of noble metal nanoclusters during oxidative polymerization (e.g., platinum nanoclusters) [17]. Resulting from the synergistic effect of different components, the conducting polymer-based composites show multifunctionalized properties and enhanced mechanical performance and processability. The structure of the composites, interfacial adhesion between the conducting polymer and other components, and the synthetic strategies would greatly affect the properties and applications of the as-obtained conducting polymerbased composites.

Although it is widely accepted that the key component of neural communications in the human body is the action potential generated at the synapses, the subsequent generation of the electrical charges plays a vital role in the signal transmission and simulation of the neural cells. The applications of ICPs in bioelectronics are quite attractive, as they are intrinsically biocompatible and mechanically soft to realize conformal matches between the electronics and tissues to reduce the foreign body response (FBR). The biocompatibility of ICPs has been extensively investigated both in vitro and in vivo. The reported results indeed demonstrated that the ICPs, especially PPy and poly(3,4-ethylenedioxythiophene) (PEDOT), possessed good biocompatibilities with cells, tissues, and organs [18-23]. Vaitkuviene et al. showed that the PPy nanoparticles synthesized by oxidative polymerization exhibited almost no cytotoxicity to the primary mouse embryonic fibroblasts, mouse hepatoma cell line, and human $\mathrm{T}$ lymphocyte Jurkat cell line at low concentrations (up to $9.7 \mu \mathrm{g} \mathrm{mL}^{-1}$ ) [19]. They also demonstrated that the PPymodified gold surface showed good biocompatibilities to support the adhesion and proliferation of mouse bone marrow-derived stem cells, similar to the bare gold and polystyrene surfaces [20]. Wang et al. reported that the PPy extraction solution showed no trace of acute and subacute toxicity, pyretogen, hemolysis, allergen, and mutagenesis. The Schwann cells in the PPy extraction solution also showed higher survival and proliferation rates compared to the saline solution; they also tested the chronic effect of the implanted PPy-coated silicone tube for bridging the transected sciatic nerve. The result showed that the PPy-coated silicone tube can improve the nerve tissue regeneration and induce only light inflammation after 6 months' implantation [21]. George et al. fabricated PPy implants doped with polystyrene sulfonate (PSS) or sodium dodecylbenzenesulfonate (NaDBS) and surgically inserted the PPy implants into the cerebral cortex of rats. Reduced gliosis and enhanced tissue-implant interactions were found on the PPy implants after either 3 weeks' or 6 weeks' implantation, which in most cases outperformed the Teflon implants as the control [22]. Moreover, Ramanaviciene et al. showed that chemically synthesized PPy nanoparticles neither demonstrated any cytotoxic effects on mouse peritoneum cells nor affected the spleen, kidney, or liver indexes and the immune-related hematological parameters of the mice. According to their observation, the PPy nanoparticles also induced no allergic response and no inflammation can be detected in the peritoneum of mice in the sixth week after the injection of PPy nanoparticles [23]. The relatively good biocompatibility of the chemically and electrochemically synthesized ICPs makes them ideal candidates for medical bionics and implantable electrodes, where high electrical conductivity, low impedance, and seamless integration of biomolecules are required to establish an integrated tissue-electrode interface $[24,25]$.

However, several drawbacks are associated with ICPs and their composites and hinder their biological applications, such as low mechanical strength, low sensitivity and selectivity, and low stability. To overcome the limitations of the ICPs, different methods and strategies have been employed and the results were carefully investigated, including (i) chemical surface modification of conducting polymers with physiologically active species; [26] (ii) blending with other nonconductive polymers with good mechanical properties, e.g., biomass-derived and biodegradable polymers such as PDLLA [27], chitosan [28], collagen [29], cellulose [13], and polysaccharide [30]; (iii) utilizing nanostructured conducting polymers such as nanoparticles, nanotube, nanowires, and nanofibers; [11] and (iv) usage of the physical and covalent surface coating technologies - the conducting polymers were used either as substrates or as coatings [13]. And the biocompatibility of these polymers in the biological tissues was also evaluated using different methods such as "in vitro" assays [31]. After all, the ICPs and their composites hold a perspective future in biological applications, especially in implantable devices and cyborg tissues.

Several review articles have been published on ICPs used in biomedical [32, 33], tissue engineering [9], and biosensors $[34,35]$. In this review, we mainly focus on the conducting polymer-based composites and devoted to summarizing their structures, properties, and applications in the biological fields, including drug delivery, neural interfacing, and tissue engineering.

\section{Conducting Polymer-Based Composites for Drug Delivery}

An efficient drug delivery system that can deliver the drug to targeted body sites and control the drug release rate precisely is able to improve the therapeutic outcomes and reduce the side effects $[36,37]$. Structuring such drug delivery systems has been long dreamed of and became more and more practical with the development of a variety of polymer-based delivery systems. From nonbiodegradable diffusion-controlled membranes [38] to biodegradable systems with a combination of diffusion and polymer matrix degradation [39], the polymer-based delivery system has shown enormous benefits in drug delivery and release. And since the 1980s, an effective and intelligent drug delivery system based on the ICPs has been developed [40, 41]. Resulting from their inherent electrical, magnetic, and optical properties, the ICPs, especially their composites, are 
expected to be used as next-generation stimuli-responsive drug delivery systems that are smart enough to adjust the release rates according to the changes of the tissue microenvironment. Various ICPs, including PPy and its derivatives, PANI [42], PEDOT, and its derivatives [43], have been used to construct new polymer-based drug delivery systems. The ICPs also showed promising biocompatibility when interfacing with different biological tissues [44], which further proved their application potential in the fabrication of drug delivery systems.

In drug delivery systems, the use of ICPs is significantly limited by the choice of dopant and the molecular weight of the loaded drug. To remove these barriers, chemical modification or physical coating methods have been used. For example, George et al. modified the surface of PPy through biotin-streptavidin coupling [45]. Due to the ability of attaching any biotin-labeled compound, streptavidin bridges the biotinylated drug and PPy (Figure 1(a)). The modified PPy composites show high stability and well-controlled drug release process. Different from the chemical surface modification, Abidian and his coworkers reported the electrochemical depositions of PPy or PEDOT nanotubes on drugloaded, electrospun biodegradable poly(L-lactide) (PLLA) or poly(lactide-co-glycolide) (PLGA) (Figure 1(b)). This nanostructured conducting polymer composites can be used for the release of dexamethasone with electrical stimulations [43]. The controlled release of dexamethasone was also reported by Wadhwa et al. [41] In their study, the prodrug of dexamethasone disodium phosphate (Figure 1(c)) was used as a dopant in PPy followed by coating PPy on the gold-coated coverslips (electrodes). The release of the dexamethasone was controlled by the cyclic voltammetry (CV) with alternating positive and negative potentials. In this study, $0.5 \mu \mathrm{g} / \mathrm{cm}^{2}$ dexamethasone can be released after each $\mathrm{CV}$ cycle and up to a total of nearly $16 \mu \mathrm{g} / \mathrm{cm}^{2}$ can be released after $30 \mathrm{CV}$ cycles (Figure 1(d)). To further enhance the drug release performance and drug-loading capacity of the ICPs, Woeppel et al. reported the usage of functionalized negatively charged porous silica nanoparticles as the dopant for PEDOT [46]. The silica nanoparticle-doped PEDOT showed significantly enhanced doxorubicin (DOX) release profiles (up to 7 -folds higher than the control) by applying electrical stimulations; different kinds of drugs, e.g., fluorescein and DNQX, have been loaded into the porous silica nanoparticle-doped PEDOT and demonstrated controllable release profiles in vivo. Biologically active dopants, such as neural growth factor and brain-derived neurotrophic factor, can also be directly incorporated into the matrices of PPy and PEDOT to promote the neurite outgrowth in vitro $[47,48]$. In summary, chemical modification of conducting polymer substrate, coating conducting polymers on the drug-loaded biodegradable polymeric substrate, or prodrug modification have been proven to be the effective methods in preparing conducting polymer composite-based drug delivery systems.

Conducting polymer-hydrogel blends were also synthesized and applied in electrochemically controlled drug delivery systems. Due to their high swelling capacity, hydrophilicity, and quick responses to external stimuli such as strain, $\mathrm{pH}$, and temperature, the hydrogels have been proposed as suit- able materials for drug delivery applications $[49,50]$. Integration of conducting polymers into the hydrogel may endow smart functions to the as-prepared composites, e.g., switchable delivery modes (on and off) and controls over the drug-releasing rate. Conducting polymers such as PANI $[51,52]$ and PPy $[53,54]$ are electropolymerized to grow inside or on the surface of different hydrogels. Semiinterpenetrating PANI-polyacrylamide hydrogel composites were prepared by Lira and de Torresi, and the electrochemically controlled release of safranin has been demonstrated in this research [52]. It was found that the release of safranin was influenced by not only the physical and chemical properties of the composites, but also the electrochemical processes. In another work, drug-loaded PPy nanoparticles were suspended in a temperature-responsive hydrogel (poly[(D,L-lactic acid)-co-(glycolic acid)]-b-poly(ethylene oxide)-b-poly-[(D,L-lactic acid)-co-(glycolic acid)] (PLGAPEG-PLGA)), which is a liquid at low temperatures but becomes a gel at body temperature, as shown in Figure 2 [54]. The composites acquire the advantages of both conducting polymers and the sol-gel transition of hydrogels, exhibiting a dual stimulus (temperature and electric field) responsiveness and can be used to trigger sensitive dosagecontrolled release of drugs. Other conducting polymerhydrogel systems such as PANI-polysaccharide hydrogels [30], PPy-alginate hydrogel [53], and chitosan-graft-PANIoxidized dextran hydrogel [55] have also been evaluated to identify their potential in drug delivery applications. Recently, near-infrared light and $\mathrm{pH}$ dual responsive drug delivery composites of PANI-biodegradable poly(ethylene glycol)-poly(E-caprolactone) (PEG5k-PCL10k) block copolymers and lecithin that are used for the controlled release of cisplatin were reported by You and his coworkers [42].

Nowadays, there is an increasing trend in the fabrication of conducting polymer-based composites for drug delivery. However, most ICPs and their composites are not biodegradable, which is considered a major drawback for the ICP-based drug delivery systems, and a postdelivery process is required to remove the drug-loaded carriers. Although several biodegradable conducting polymer composites have been reported [56], it is still a challenge to achieve both high conductivity and appropriate biodegradability. Meanwhile, conducting polymer composites that can response to dual or more stimuli are expected to find widespread applications in drug delivery for their more precisely controlled release rate.

\section{Conducting Polymer-Based Composite Electrodes for Neural Interfacing}

The nervous system is of great complexity due to the thousands of networks of neurons and supporting cells, which work synchronously to process external and internal cues that result in movements, emotions, sight, speech, and hearing. It is important for knowing the mechanism of the neural network and treating some neural disorders to understand the nervous system, which has engaged the interests of scientists. The neural interface serves as a medium between electrical devices and neural tissues. In other words, the neural 


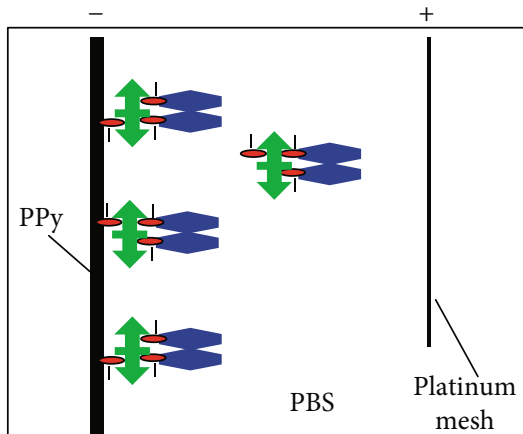

0- Biotin
Biotinylated
NGF
Atreptavidin

(a)

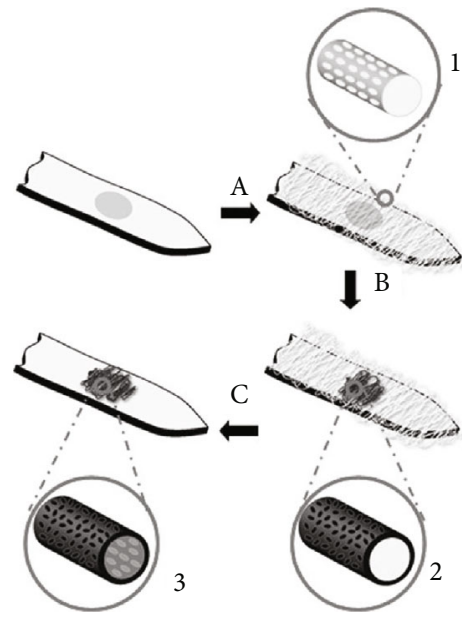

(b)<smiles>CCC1(O)C(C)CC2C3CCC4=CC(=O)C=CC4(C)C31C(O)CC2(C)C(=O)COP(=O)(O[NH3+])O[Na]</smiles>

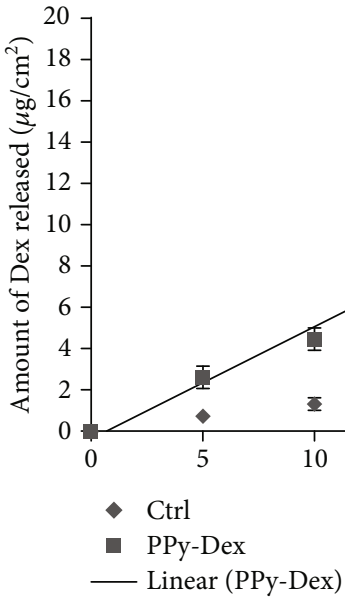

(c)

(d)

FIGURE 1: Schematic illustrations of the modification of conducting polymers and prodrug for drug delivery systems: (a) release of the biotin from the PPy surface; [45] (b) surface modification of neural microelectrodes to create nanotubular PEDOT; [43] (c) chemical structures of the dexamethasone disodium phosphate (Dex); [41] (d) release profile of Dex from the polymer-drug-coated electrodes [41].

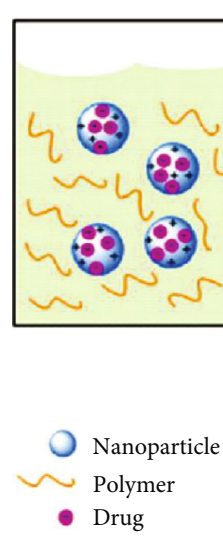

(a)

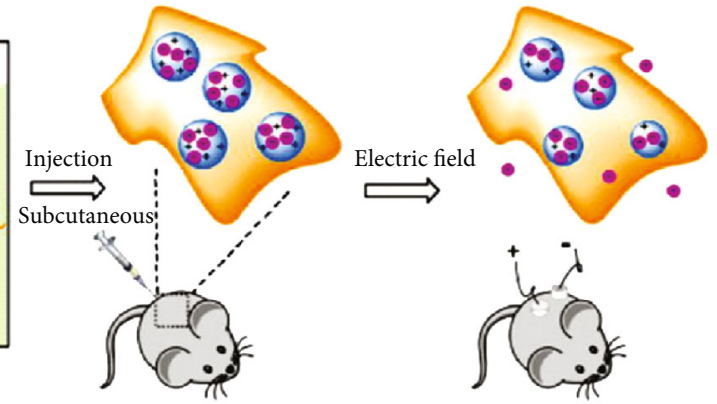

(b)

(c)

FIGURE 2: General scheme for the application of conducting polymer-hydrogel system. (a) The nanoparticle polymer solution is (b) subcutaneously injected into a mouse, followed by (c) application of a DC electric field to induce release of the drug cargo inside the nanoparticles [54]. 
interface is a tool to interface between biological and electronic systems, allowing for monitoring and manipulation of neural circuits that underpin both normal and diseased states. The information of nervous networks and their responses to the bioactive materials have been effectively provided by the neural electrodes. When implanted into the brain, neural electrodes can record the signal from the neuron for studying the mechanism of the brain and also can give the degenerated tissues in the brain effective stimulations to heal neurological diseases such as Parkinson's disease, epilepsy, deafness, blindness, and movement disorders. Therefore, it is vital for neural research to have reliable signals taken from the brain for the accurate analysis of the neural system and selective stimulations. For clinical diagnosis, it requires a neural electrode with a high conductivity, low impedance, and good biocompatibility. The traditional neural electrodes are mostly composed of noble metals and may not be competent for long-term implantations and signal recording due to the mechanical mismatch between the stiff metal electrode $\left(E_{\text {metal }}=74-530 \mathrm{GPa}\right.$ [57]) and soft neural tissue $\left(E_{\text {brain }}=2.1-3.7 \mathrm{kPa}\right.$ [58], $E_{\text {spinal cord }}=$ $3-6.3 \mathrm{kPa}[59]$, and $\left.E_{\text {peripheral nerve }}=576-840 \mathrm{kPa}[60,61]\right)$. The mechanical mismatch between the electrodes and neural tissues may induce significant FBR at the electrodeneural tissue interface, resulting in the malfunction of both the electrodes and the surrounding tissues. Current research is aimed at producing stable, small, and high-density microelectrodes in order to improve the recording and stimulating selectivity for both in vitro and in vivo applications. To carry out implantation of neural electrodes precisely and selectively and reduce the unwanted damage of neural tissues, the fabrication of neural electrodes with a smaller geometrical size, e.g., microelectrodes, has been widely adapted. However, the small geometrical sizes also limit the electrical functions of the microelectrodes, and increased impedance and reduced charge injection properties are associated with the microelectrodes compared to the bulky ones. To address this issue, the geometric surface of the microelectrodes can be miniaturized to form conductive, porous, and biocompatible nanostructures [62].

Strategies to improve the electrochemical surface area whilst maintaining the desired geometric area include the development of rough microelectrodes and microelectrode coatings onto traditional planar microelectrode materials such as gold and platinum. The metal electrode (gold, platinum, and silver) was coated by conducting materials through chemical and electrochemical deposition. The conducting materials including graphene and graphene oxide (GO) [63], iridium oxide $\left(\mathrm{IrO}_{\mathrm{x}}\right)$ [64], carbon nanotubes (CNTs) [65], and ICPs [66]. ICPs with high conductivity, excellent charge transport capacity, and good biocompatibility were speculated as promising electrode materials for nextgeneration neural electronics with reduced neural interfacing impedance. Few researches focused on the way to coat ICPs like PPy [67], PANI [68], and PEDOT [69] on the surface of metallic microelectrodes to enhance their electrochemical properties, which is considered simple but effective. Compared to the bare metal electrodes, the impedance of the modified neural electrodes became smaller due to the increased electrochemical surface area after the deposition of ICPs. For example, Zhou et al. fabricated a PEDOT and multiwall carbon nanotube (MWCNT) composited thin film and coated it onto platinum microelectrodes by different electrochemical deposition methods, i.e., potentiostatic and galvanostatic [70]. Compared to the PEDOT/MWCNT coating formed by the potentiostatic method, the PEDOT/MWCNT film from the galvanostatic method showed a porous morphology composed of tangled rods with a smaller average diameter $(\sim 50 \mathrm{~nm})$. The impedances of the PEDOT/MWCNT-coated microelectrodes from the two methods exhibited almost two orders of magnitude lower than the bare platinum microelectrode at $1 \mathrm{kHz}$. The impedance at $1 \mathrm{kHz}$ is a typical parameter to evaluate the functions of the neural electrodes, as it correlates to power consumption for the electrical stimulation of the neural tissues and the neuronal action potentials. However, the stability of the ICP coating on the microelectrodes is also critical for achieving chronic monitoring of the neural tissues. Few researches have focused on solving these problems by doping inorganic and organic substances into the ICP coating to enhance the adhesion between the ICP films and the substrates and also to enhance the biocompatibility of the electrodes corresponding to the neural tissues. For example, Bodart et al. polymerized PEDOT:tetrafluoroborate (PEDOT: $\left.\mathrm{BF}_{4}\right)$ onto the platinum neural electrode by using three different solvents, i.e., propylene carbonate (PC), acetonitrile (ACN), and water [71]. The mechanical and electrochemical stabilities of the as-obtained composited electrodes were subsequently analyzed. The result indicated that $\mathrm{PEDOT}: \mathrm{BF}_{4}$ coatings deposited in organic solvents on platinum-iridium (PtIr) microelectrodes remained physically stable after $5 \mathrm{~min}$ of sonication and retained more than $80 \%$ of their charge storage capacity (CSC), while coatings deposited in deionized water detached from the PtIr microelectrodes after 2-3 min of sonication. After soaking in the PBS ( $\mathrm{pH}=7.4)$ solution for two weeks, the impedances of PEDOT-coated microelectrodes obtained in PC and ACN were increased but still considerably lower than the bare PtIr electrode. They concluded that the PtIr electrode coated by the PEDOT:BF ${ }_{4}$ film from the organic solvents showed better stability under ultrasonication, PBS soaking, and steam sterilization than the PEDOT:BF ${ }_{4}$ coating from water. Cui et al. electrochemically polymerized PPy and a biomolecule (a silk-like polymer having fibronectin fragments (SLPF) and nonapeptide CDPGYIGSR) onto the gold electrode sites of a neural probe [72]. Apart from better performance in electrical properties than the uncoated electrodes, more cells $(1.25 \pm 0.6$ cells per site) were grown on the PPy/SLPF-coated gold electrodes than on the uncoated ones. They also found that human neuroblastoma cells preferred to seed onto the PPy/CDPGYIGSR-modified electrode selectively compared to the electrode coated with $\mathrm{PPy} / \mathrm{CH}_{3-}$ $\mathrm{COO}^{-}$(Figure 3 ). It indicated that by doping different biomolecules in the conducting polymer coating deposited on the neural electrode, the cell behaviors on the neural electrodes can be mediated to promote the formation of integrated tissue-electronic interfaces.

To further decrease the mechanical mismatch between the electrodes and tissues, and achieve the so-called conformal 


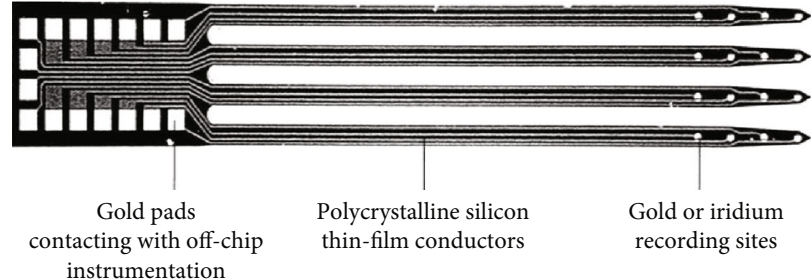

(a)

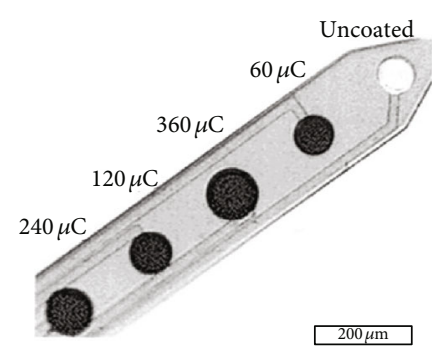

(b)

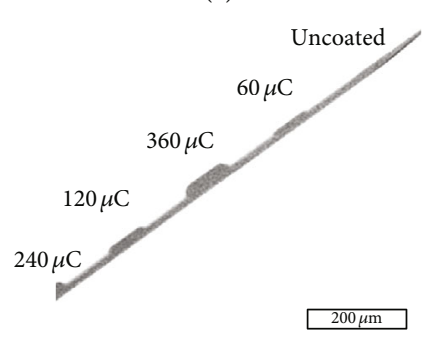

(c)

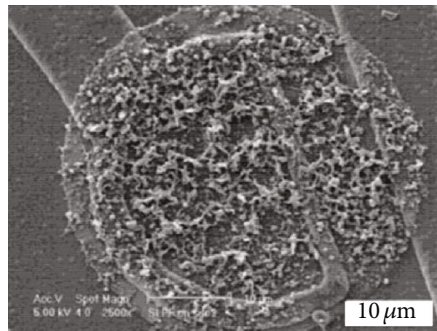

(e)

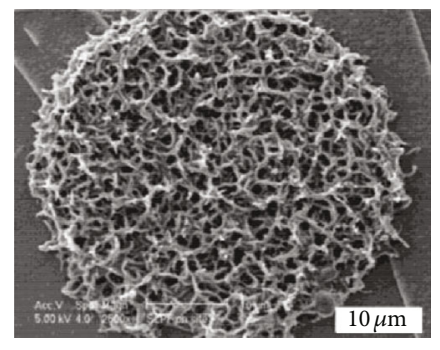

(f)

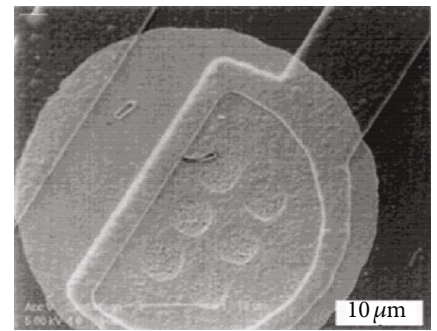

(d)

FIgURe 3: (a) Micromachined metal/silicon-based neural recording probe. (b, c) Optical micrographs of a PPy/PSS-coated 5-channel neural probe. (b) is the top view of the probe, and (c) is the side view from which the thickness of the film can be estimated. The area of each metal electrode is $3900 \mu \mathrm{m}^{2}$, and the thickness of the probe is $15 \mu \mathrm{m}$. By varying the deposition charge, the amount of material deposited can be precisely controlled. (d-g) SEM images of PPy/SLPF-coated electrode sites. From (d) to (g), the deposition time increased, corresponding to a total charge passed of (d) $0 \mu \mathrm{C}$, (e) $1 \mu \mathrm{C}$, (f) $4 \mu \mathrm{C}$, and (g) $10 \mu \mathrm{C}$. The area of the uncoated electrode site is $1250 \mu \mathrm{m}^{2}$ [72].

electronic-tissue interface, soft substrates such as Parylene C [73], polydimethylsiloxane (PDMS) [74], SU-8 [75], polyimide (PI) [76], and silk fibroin [77] can be introduced to fabricate flexible and highly conductive neural electrodes. Compared to the metal substrate, PI, SU-8, Parylene C, and PDMS acquire much smaller Young's moduli of $8.45 \mathrm{GPa}$, 5.6 GPa, 4.0 GPa, and 1.0 MPa, respectively. These materials could be utilized to fabricate flexible neural electrodes of different shapes and sizes with improved conformal matching for minimally invasive implantation. For example, Heo et al. fabricated a PI nanofiber- (NF-) based neural interface with a high flexibility and permeability for stable neural signal recording [78]. The neural interface they designed consisted of a PI NF substrate, electronic connection pads composed of silver nanoparticles, and PEDOT: poly(styrenesulfonate) (PEDOT:PSS) as the advanced conductive layer (Figure 4). They demonstrated that the as-obtained NFbased nerve electrode showed electrochemical properties superior to those of conventional cuff electrodes. And it was able to record neural signals for a long period of time, i.e., after implantation for 12 weeks.

\section{Conductive Polymer-Based Electronics for Minimally Invasive Electronics}

The ICPs, such as PPy [79] and PEDOT [80], can be used as the electrode materials for flexible and stretchable electronics. Their major advantages include biocompatibility and large surface area, resulting in higher in vivo charge transfer capacities [81]. Various biomolecules, such as enzyme, DNA, growth factor, and antibody, can be readily incorporated into the ICPs through electrochemical polymerization as the stimuli to enhance the acquisition of biological cues [82-85]. On the other hand, the CP-based stretchable electrodes for engineered tissues can also be realized by incorporating metal-based or carbon-based nanoparticles into elastomers, such as PDMS or styrene-butadiene-styrene (SBS) [86]. Another way to make CP-based flexible and stretchable electronics is to infuse liquid metal alloys inside microfluidic channels, e.g., filling the eutectic galliumindium in the PDMS channels.

Bioresorbable macromolecules and polymers can be used as the substrates for the fabrication of $\mathrm{CP}$-based 


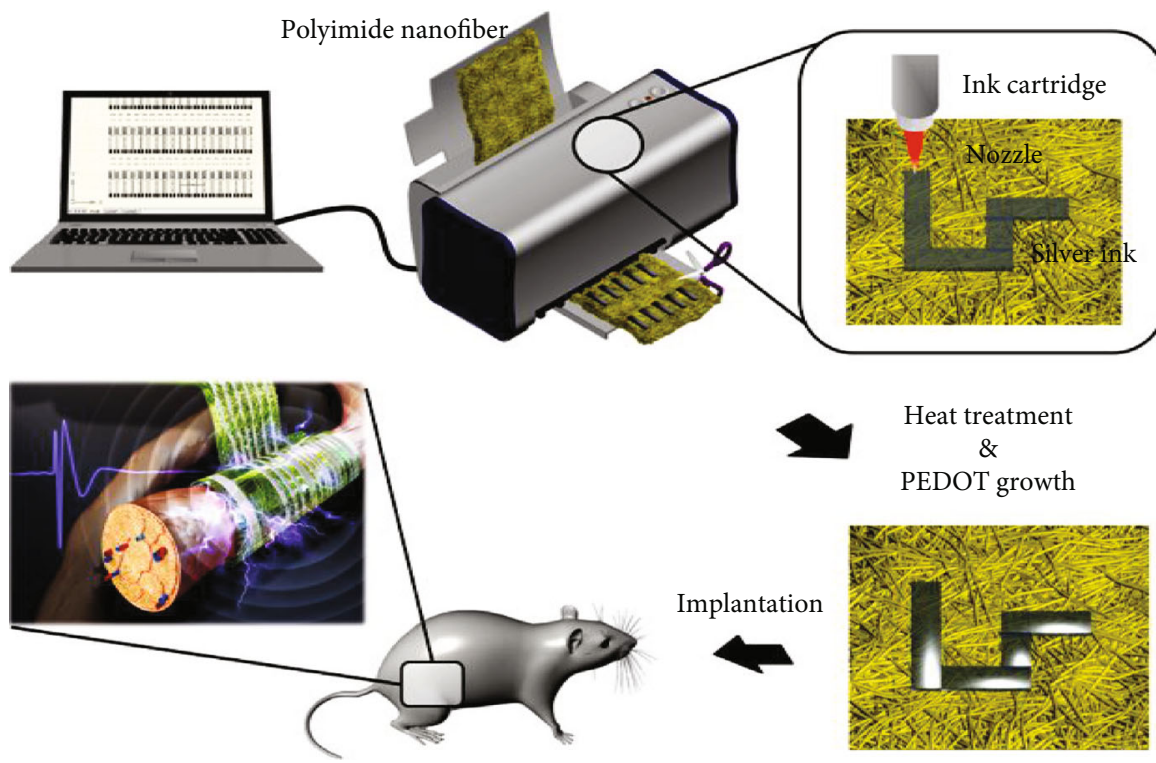

(a)

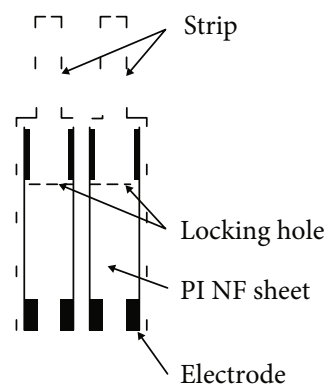

(b)

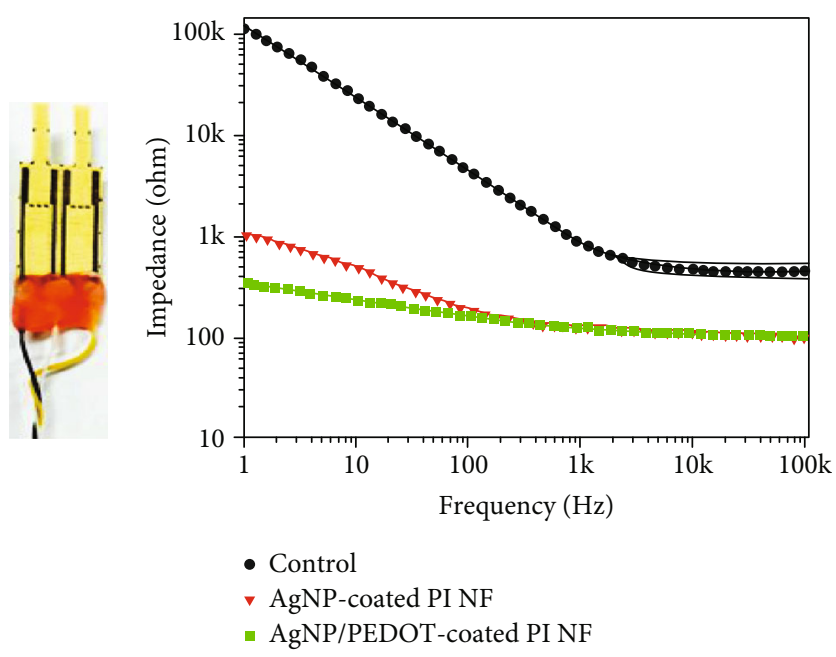

(c)

(d)

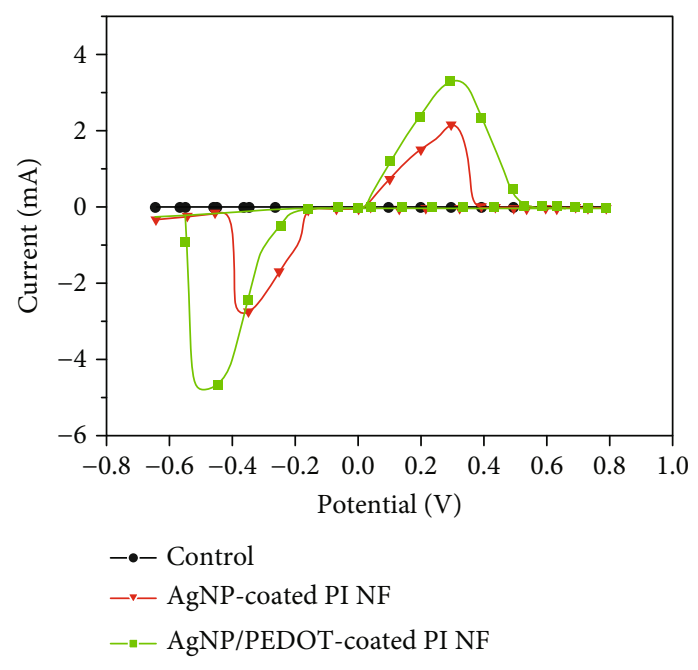

(e)

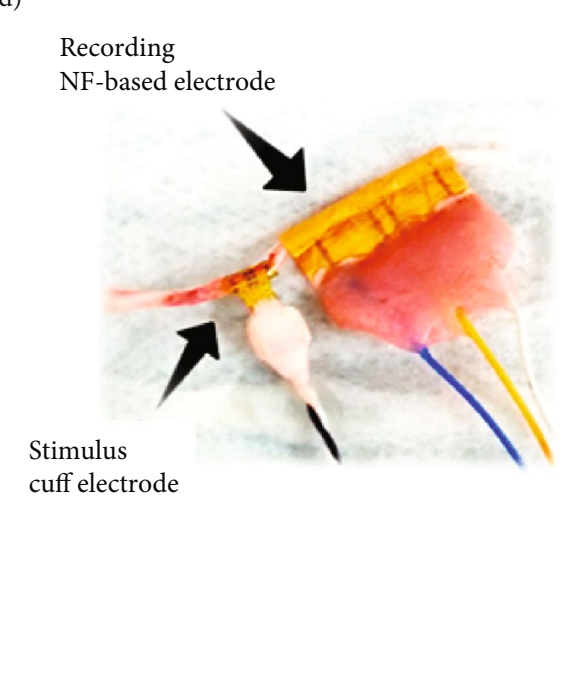

(f)

Figure 4: Continued. 


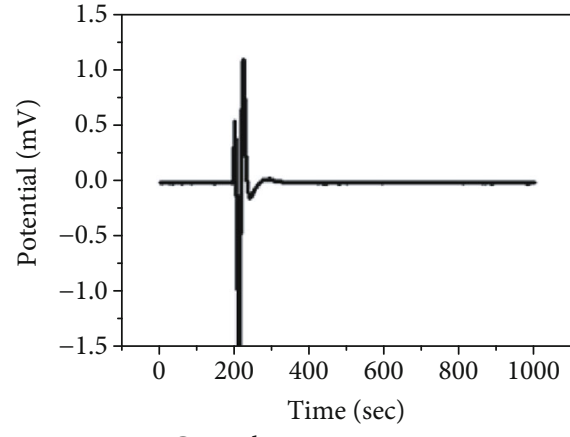

(g)

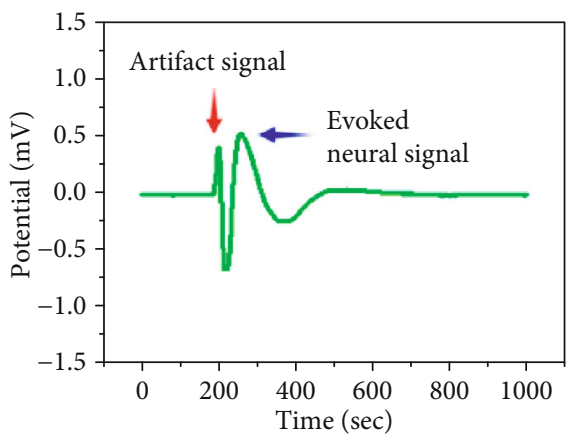

- AgNP/PEDOT-coated PI NF

(i)

Day 0

4 weeks
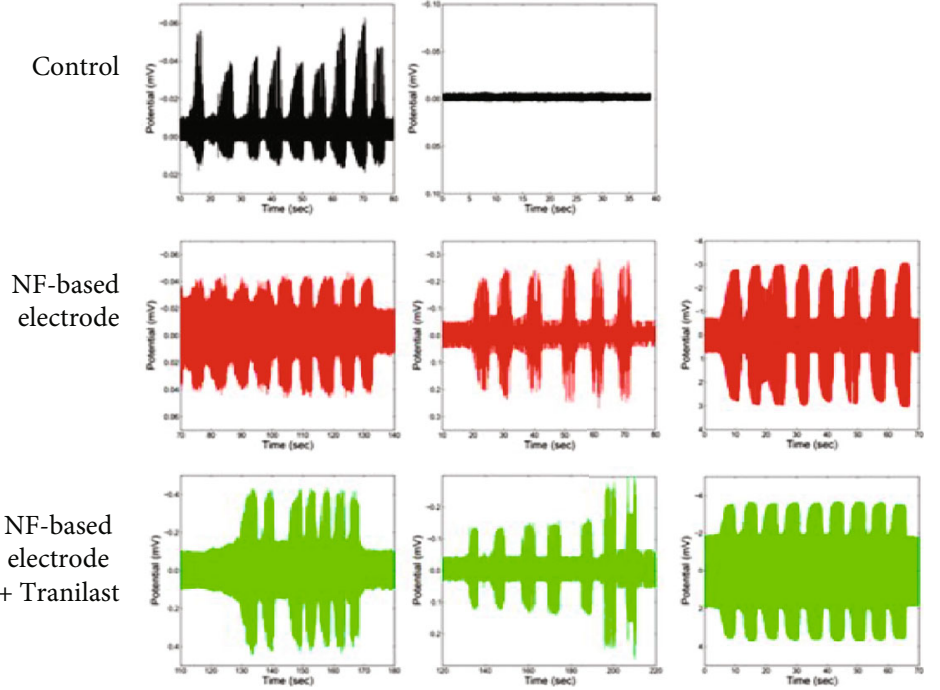

(k)

\section{2 weeks}

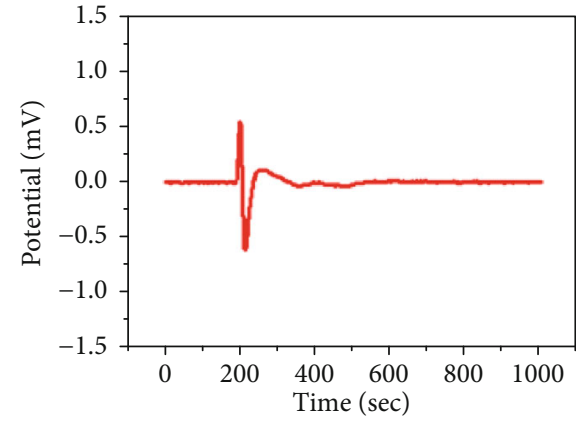

— AgNP-coated PI NF

(h)

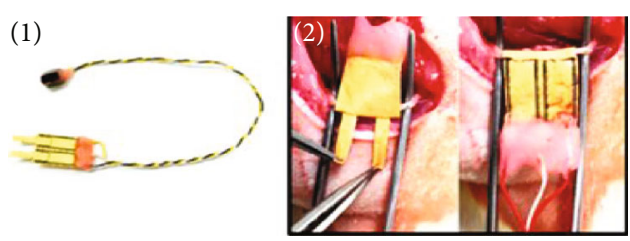

(j)

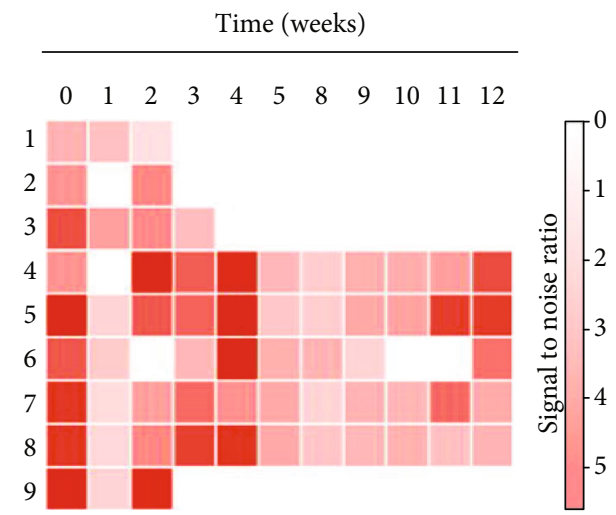

(l)

Figure 4: (a) Scheme of the fabrication process using an inkjet printing system. (b) Concept of preproduction design and (c) image of the fabricated NF-based neural electrode. Electric wire cables were bonded to the end of the electrode pads using conductive silver epoxy and were covered with bone cement. (d) Electrochemical impedance spectrum over a frequency range of 1-105 Hz. (e) Cyclic voltammograms with a scan range of -0.7 to $+0.8 \mathrm{~V}$ at a scan rate of $100 \mathrm{mV} / \mathrm{s}$. (f) Image of a wrapped stimulus cuff electrode and a recording NF-based electrode. Acute ex vivo recordings obtained using (g) a control electrode, (h) AgNP-coated PI NF electrode, and (i) AgNP/PEDOTcoated PI NF electrode. (j) Electrical packaging electrode (1) and implanted electrode (2). The NF-based electrode is wrapped around the sciatic nerve of a rat. $(\mathrm{k})$ Representative neural signal recording obtained from sciatic nerve tissue over a period of 12 weeks using a control electrode, an NF-based electrode, and an NF-based electrode soaked with Tranilast. (l) Simultaneous mapping of the SNR of an NF-based electrode with and without Tranilast [78]. 
bioelectronics. The utilization of bioresorbable materials to construct the bioresorbable electronics can effectively eliminate the adverse effects of long-term implantation such as the FRB and unfavorable biofilm formation [87]. The substrate layer of bioresorbable electronics can be fabricated by natural or synthetic polymers with tunable degradation properties. For example, the natural protein silk fibroin [88] which can be degraded into natural amino acids by enzymes in vivo. Alternatively, bioresorbable synthetic polymers, such as PLGA, can also be used. The advantages of the natural polymers include higher biocompatibility, reduced FBR, and higher mechanical strengths. Disadvantages include higher cost and lower processability. Compared with natural polymers, the synthetic polymers show the advantages including controllable degradation rate and low cost [89].

The conductive components of the CP-based bioelectronics, such as semiconductors and dielectrics, can be made of silicon and silicon dioxide, which would degrade into nontoxic $\mathrm{Si}(\mathrm{OH})_{4}$ in physiological solutions, and the dissolution rate is dependent on the thickness. For the metallic conductors, the biocompatibilities of gold and platinum are good, but they are not degradable. Magnesium and zinc can dissolve rapidly, and the degradation products are magnesium hydroxide and zinc oxide, which can be easily removed by the human body [90]. Tungsten, molybdenum, and iron can also be dissolved, but the dissolution rates are slower, and the degradation products are iron oxides or hydroxides and tungsten and molybdenum oxides [88]. Therefore, the metal conductors may need to be selected according to the life expectancy of the bioresorbable electronics. Zinc and magnesium are suitable for short-term implantation, while tungsten, molybdenum, and iron are suitable for long-term implantation [91].

The flexible polymeric substrates and conductive materials can be integrated to fabricate $\mathrm{CP}$-based bioresorbable electronics in various structures and configurations to achieve minimally invasive implantations, e.g., the injectable bioelectronics. Typical structures of the injectable bioelectronics are cylindrical, consisting of a microchip and integrated electronic components such as semiconducting electronic components, solenoid microcoils, and hybrid charge storage capacitors. All the electronic components of the injectable bioelectronics are combined in a thin film of an integrated circuit chip. Metal electrodes are extended from both ends of the device, acting as the stimulation electrodes to activate the nearby nerves or muscle motions [92]. With the development of injectable bioelectronics, reticular electronic implants were found to have excellent mechanical and structural properties and were widely used in the neural research, e.g., the single-neuron chronic recording for the retina in awake mice [93] and seamless syringe-injectable mesh electronics with minimal chronic immune response for brain monitoring [94]. Mesh-shape flexible bioelectronics provide new solutions for the signal recording and stimulation of brain tissues during implantation, and they can avoid problems associated with traditional rigid wire-shape electrodes such as mechanical mismatch and FBR [93-99]. The unique macroporous morphology with submicrometer thickness and cell-scale-wide mesh elements may realize the stable and seamless tissue integration by virtue of their unique mechanical and structural properties [94, 95, 97]. Previous studies on the mesh-shape electronics also demonstrated stable chronic recording at the single-neuron level for at least eight months [91].

Key points related to the structural design of standard mesh electronics are highlighted in Figures 5(a) and 5(b) [100]. Periodic unit structures are constructed for the standard mesh electronic probes (Figures 5(a) and 5(b)). The unit cell of the mesh consists of longitudinal elements parallel to the injection direction and transverse elements oriented at an angle $\alpha$ of $45^{\circ}$ relative to the longitudinal direction (Figure 5(b)) [94, 96-99]. The longitudinal elements may have composite structures, consisting of gold interconnects sandwiched between two layers of the biocompatible photoresist SU-8 [101]. One end of the longitudinal element is connected to a sensor or stimulator such as a metal electrode [94-98, 102] or nanowire transistor [95, $102,103]$, and the other end is connected to an input/output (I/O) pad. The transverse elements may consist of two SU-8 layers with a total thickness of approximately $800 \mathrm{~nm}$ and a width of $20 \mu \mathrm{m}$ [95]. The transverse elements determine the transverse bending stiffness of the overall mesh structure, and correspondingly, it also determines the rolling degree of grid probes when they are loaded into the needle. After all, both longitudinal and transverse elements contribute to the longitudinal bending stiffness of the mesh probe, and a sufficient longitudinal bending stiffness may maintain the overall linear structure of the mesh electronics during injection $[95,96]$.

Besides the mesh-like structure, biomimetic neuron-like structures for implantable bioelectronics were also proposed by Yang et al. [104] The neuron-like electronic unit (NeuE) is structurally and mechanically mimicking a neuron at the subcellular level. The photoresists of SU-8 and S1805 were used as the polymeric phase while chromium, gold, and platinum were used as the conductive phase of the NeuE. The sizes of the metal recording blocks of NeuE also match those of the targeted neural cells. The interconnects of the neuronlike structure have similar flexibility to the axons. And the thin polymer insulating layer of the structure is also analogous to the myelin sheath. Both of these features promote in the propagation of electrical signals from the neural cells to the electrodes. The NeuE can also be assembled into an open three-dimensional neuron-like electronic network with structures and morphologies mimicking the natural neural network. However, the properties and functions of the implantable bioelectronics are closely related to their designed structures. In recent studies, mesh-like minimally invasive electronics are often reported to have ultraflexible structures so that they can on the one hand be delivered by capillary needles and on the other hand be perfectly adapted to the nonplanar surface of the brain tissues. The ultraflexible mesh electronics comprising the SU-8 polymeric layer and metallic electrodes can minimize the accumulation of astrocytes and glial cells $[94,95]$. In the meantime, it can also reduce the glial scarring (Figure 6(a)), resulting in little or no chronic immune responses in the brain [94, 105]. 
(a)

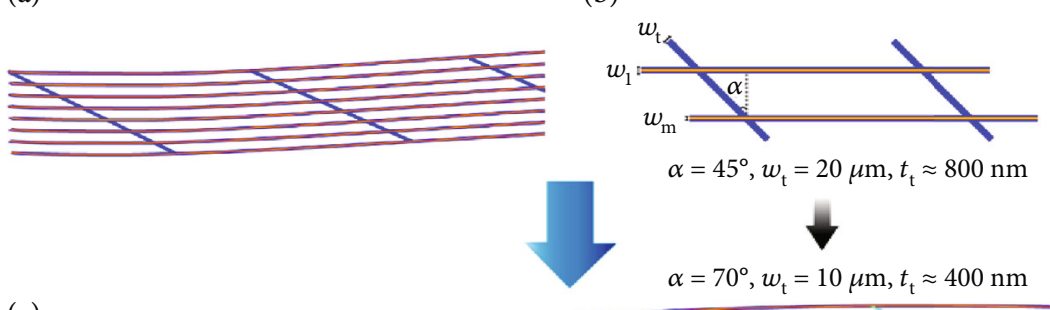

(c) (b)

(d)
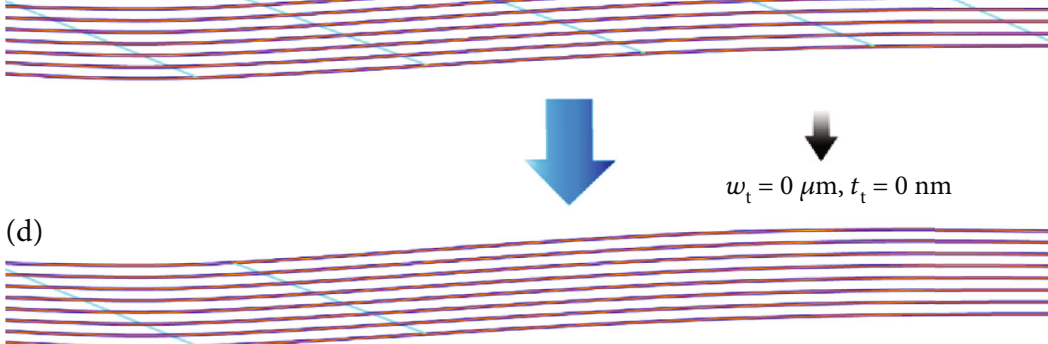

Figure 5: Structural design of ultraflexible syringe-injectable mesh electronics. (a) Schematic of a conventional mesh electronic probe. (b) Schematic of the mesh electronic unit cell, highlighting key probe design parameters, including the angle between longitudinal and transverse elements $(\alpha)$, the longitudinal element width $\left(w_{1}\right)$, the transverse element width $\left(w_{\mathrm{t}}\right)$, the longitudinal interconnect metal width $\left(w_{\mathrm{m}}\right)$, and the transverse element thickness $\left(t_{\mathrm{t}}\right)$. For the standard mesh $\left(\alpha=45^{\circ}, w_{1}=20 \mu \mathrm{m}, w_{\mathrm{t}}=20 \mu \mathrm{m}, w_{\mathrm{m}}=10 \mu \mathrm{m}\right.$, and $\left.t_{\mathrm{t}} \approx 800 \mathrm{~nm}\right)$. (c) Schematic of ultraflexible 2D mesh electronics to facilitate loading and injection using reduced diameter needles and injection volumes, where $\alpha=70^{\circ}, w_{\mathrm{t}}=10 \mu \mathrm{m}$, and $t_{\mathrm{t}} \approx 400 \mathrm{~nm}$; parameters for the longitudinal elements are the same as in the standard design highlighted in panel (b). (d) Schematic of ultraflexible 1D mesh electronics with transverse element removal in the implantation site, i.e., $w_{\mathrm{t}}$ and $t_{\mathrm{t}}$ equal to zero, and the parameters for the longitudinal elements and transverse elements external to the implantation region are the same as the 2D probes highlighted in panel (c). Orange lines represent the gold interconnects, dark blue lines represent two SU-8 layers for interconnect insulation, and the cyan line represents a single SU-8 layer [100].

The implant process was conducted by ultra-small-size needles; therefore, minimal damage was induced at the implant site $[93,94]$. To inject the device into targeted tissues, the ultraflexible mesh-shape electronics are preloaded in a glass capillary needle, then are injected to the targeted site, and the mesh electronics are unfolded (Figure 6(b)) [93, 95]. The match between the injection rate of the mesh electronics and the retraction rate of the needle after injection has been proven to be the key factor to deliver the mesh electrons to the targeted tissue with an unfolded conformation (Figure 6(c)) [96]. On the other hand, the use of needles and probes with smaller sizes can further reduce the damage applied to the tissues and maintain the original vascular and cellular structures during the injection process. Several methods have been reported to reduce the probe size, e.g., the utilizations of ultraflexible two-dimensional (2D) and one-dimensional (1D) probes have been proven to be effective in reducing the transverse bending stiffness of the electronics, and they can be injected through the glass needles with an inner diameter as small as $100 \mu \mathrm{m}$ and an outer diameter of $170 \mu \mathrm{m}$ [100]. In another study, the devices made by the nanoelectronic thread (NET) were reported to obtain a minuscule size [105]. The epoxy-based SU-8 was used as the polymeric phase while gold and platinum were used as the conductive interconnects and electrodes for NET. The NET-50 showed a total thickness of $1 \mu \mathrm{m}$ and an average width of $50 \mu \mathrm{m}$, and the NET-10 showed a cross-sectional area of $10 \mu \mathrm{m} \times 1.5 \mu \mathrm{m}$. Due to the extremely small thickness, the bending stiffness of NET-50 and NET-10 is much lower than those of the typical silicon, carbon fiber, PI, and Parylene C probes (Figure 6(d)). Recently, Liu et al. showed that the longitudinal and transverse bending stiffness of the mesh-shape electronics, $D_{\mathrm{L}}$ and $D_{\mathrm{T}}$, was dependent on the angle between the longitudinal and transverse elements ( $\alpha$ shown in Figure 5(b)) [95]. When $\alpha=45^{\circ}, D_{\mathrm{L}}$ and $D_{\mathrm{T}}$ were $\sim 2.5 \times 10^{-3} \mathrm{nN} \cdot \mathrm{m}$ and $\sim 10^{-2} \mathrm{nN} \cdot \mathrm{m}$, respectively. And the bending stiffness of the injected mesh electronics can be reduced to $0.087 \mathrm{nN} \cdot \mathrm{m}$. The bending stiffness was further reduced to $10^{-15} \mathrm{~N} \cdot \mathrm{m}^{2}$ by utilizing the NET structures which realized the glial scar-free neural integration [105]. Moreover, the bending stiffness of the biomimetic neuron-like electronics decreased 5-20 times as compared to the other state-of-the-art mesh electronic designs, and both a structurally and functionally stable tissue-electronic interface can be formed after implantation [104].

The electronic properties such as stable impedance and large signal-to-noise ratio (SNR) are also critical to achieve a highly integrated tissue-electronic interface for chronic and real-time monitoring. For the NET probes, the impedance was around $750 \mathrm{k} \Omega$ after implantation and decreased to around $600 \mathrm{k} \Omega$ in the first 1.5 months. The impedance was then observed to remain stable for the next 2.5 months accompanied with low noise levels (Figure 6(e)) [105]. Sortable single-unit action potentials (APs) with stable average amplitude and SNR can also be obtained by the NET probes throughout the experimental period of 4 months (Figure 6(f)). On the other hand, the mesh electronics showed higher biocompatibilities as compared to the flexible 


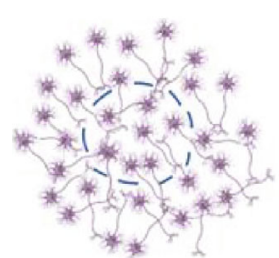

Mesh

(a)

(1)

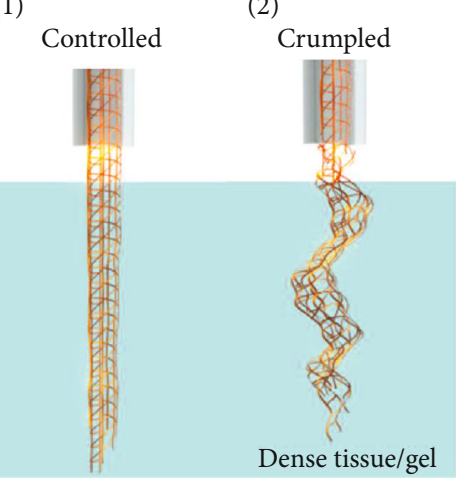

(c)

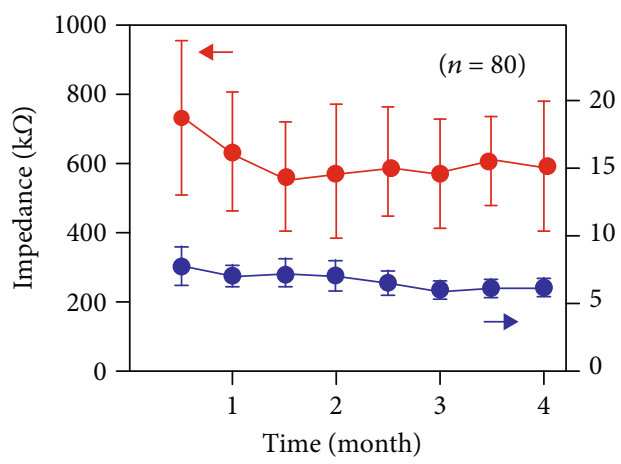

(e)

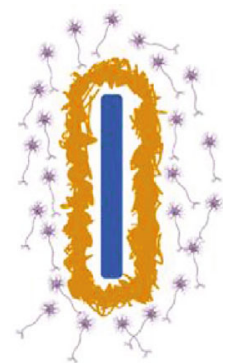

Flexible thin-film

(2) Dense tissue/gel

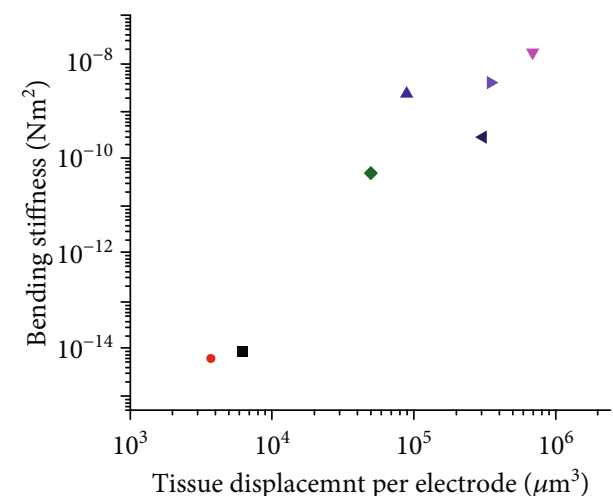

- NET-50

- NET-10

4 Typical silicon probe

- Typical microwire

- Carbon fiber probe

Polyimide probe

- Parylene C probe

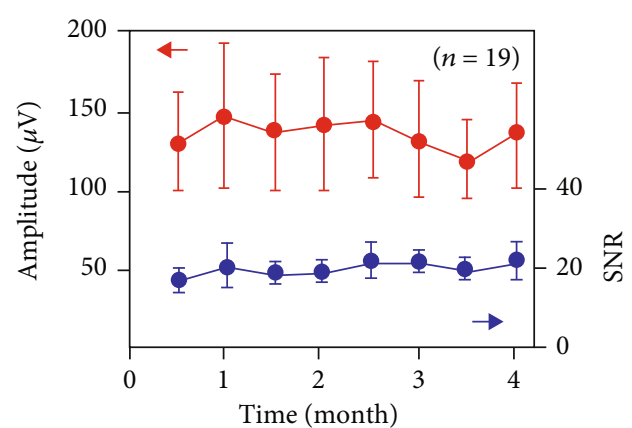

(f)

Figure 6: Continued. 


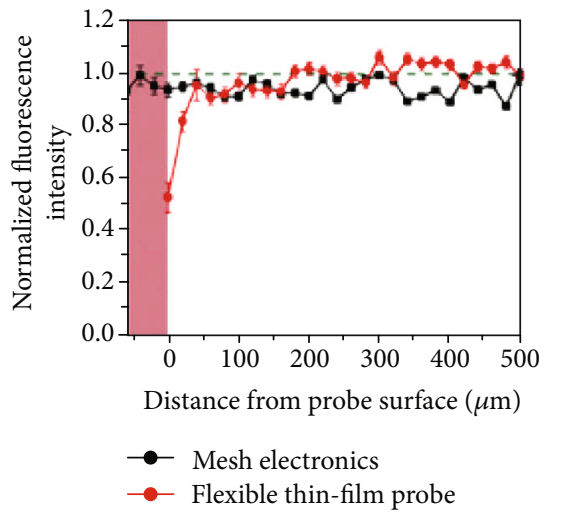

(g)

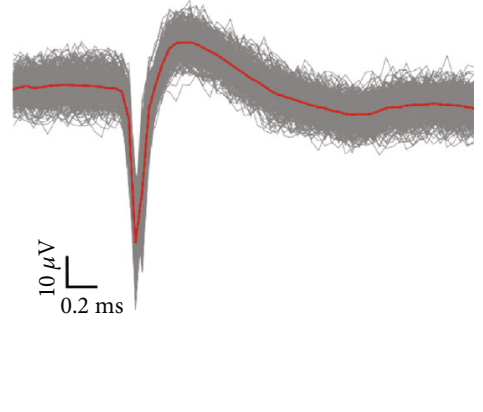

(h)

FIgURE 6: (a) Thin-film electronics may induce the glial scar formation while the mesh electronics may not. The blue lines represent different types of electronics (mesh and thin film), the neurons are in purple, and the glial scars are in yellow [94]. (b) Schematic showing a mesh electronic that is injected into the tissue and extended [95]. (c) Mesh electronics injected by the balanced FoV approach was extended (1). And a mismatched injection may result in a wrinkled mesh (2) [96]. (d) A comparison between the bending stiffness of the NETstructured probes and other typical fiber-based probes [105]. (e) Stable impedance (red) and low noise level (blue) were demonstrated by the NET-structured probes during a long-term recording process ( 4 months) [105]. (f) Stable average amplitude (red) and large SNR (blue) for the chronic recording of single-unit APs were also observed on the NET probes [105]. (g) Stable fluorescence intensity of neuron somata can be obtained near the surface of the mesh electronics as compared to the flexible thin-film probes [94]. (h) Single-unit neural recording from one channel of the mesh electronics; the average potential waveform (red) showed characteristic average duration of $2 \mathrm{~ms}$ and peak-to-peak amplitude of $70 \mu \mathrm{V}$ of the single-unit APs [95].

thin-film probes according to the immunohistochemical characterization, as the fluorescence intensity corresponding to the neuron somata was much higher near the surface of the mesh electronics (Figure 6(g)), and the intensities of astrocytes and microglia were much lower [94, 97, 104]. Controlled injection of the mesh electronics has also been realized by a stereotaxic surgery station equipped with a syringe pump, and a spatial precision of ca. $20 \mu \mathrm{m}$ has been achieved. And subsequently, 100\% multichannel input/output $(\mathrm{I} / \mathrm{O})$ connectivity between the mesh electronics and a flexible cable device can be achieved by using a computercontrolled conductive ink printing technique [96].

The CP-based injectable electronics with minimal invasiveness have great potential for neural study and brain recording such as single-unit neural AP recording [95], brain mapping [97, 106], and biochemical sensing [107] which may promote the establishment of a next-generation brainmachine interface for both fundamental neuroscience and therapies [108]. By maximally reducing the tissue damage during implantation and minimizing the chronic immunoresponses, the CP-based injectable electronics can serve as a long-term monitoring platform in vivo [109] and can retrieve the physiological signals from the deep brain regions [110]. The CP-based injectable electronics can also integrate with the wireless devices for the applications of wireless optogenetics [111].

\section{Conclusions and Future Work}

Upon integrating the flexibilities of polymers and conductivities of metals, the CP-based composites show promising applications for the in vivo therapeutics and implantable electronics, including drug delivery, neural interfacing, and injectable electronics. The ICPs can be utilized as the biocompatible, tunable, and controllable advanced delivery platforms for the in vivo targeted transport of the negatively charged drug molecules. And they can also be integrated with other functional materials such as metallic electrodes and hydrogels to form multifunctional composites for tissue monitoring, stimulation, and drug delivery. Highperformance neural recording electrodes with significantly enhanced biocompatibility and sensitivity can be fabricated by coating the nanostructured ICPs onto the metallic electrodes. Flexible electronics which conformally comply with the epidermal layer of tissues can also be obtained by coating the ICPs on polymeric substrates using various techniques, such as photolithography, inkjet printing, and electrodeposition. On the other hand, ultraflexible CP-based composites can be obtained by integrating the polymeric photoresists with metallic electrodes in various structures and configurations, such as the mesh- and thread-like shapes. The ultraflexible CP-based composites can be further utilized as the injectable electronics for minimally invasive implantation and show high performance in chronic recording, neural monitoring, and single-neuron-level brain mapping. To further transform the lab-based technique into therapeutic applications, the mechanical property and chemical stability of the ICPs may need to be improved for drug delivery. The chronic performance of the ICPs and the patterning precision of the printing electronics may need to be further improved for the flexible electronics. Finally, concerns over the biocompatibility of the CP-based injectable electronics also arise since highly cross-linked nondegradable SU-8 and metallic chromium with biological toxicity are used. 


\section{Conflicts of Interest}

The authors declare no conflict of interests.

\section{Acknowledgments}

The authors gratefully acknowledge the financial supports from Sun Yat-sen University, the China Postdoctoral Foundation (2018M633230), the National Natural Science Foundation of China (81801851), and the Guangdong Science and Technology Department (2018A030310075).

\section{References}

[1] G. Inzelt, Conducting polymers: a new era in electrochemistry, Springer Science \& Business Media, 2012.

[2] S. S. Shinde and J. A. Kher, "A review on polyaniline and its noble metal composites," International Journal of Innovative Research in Science, Engineering and Technology, vol. 3, no. 10, pp. 16570-16576, 2014.

[3] L. Pan, H. Qiu, C. Dou et al., "Conducting polymer nanostructures: template synthesis and applications in energy storage," International Journal of Molecular Sciences, vol. 11, no. 7, pp. 2636-2657, 2010.

[4] C. Peng, S. Zhang, D. Jewell, and G. Z. Chen, "Carbon nanotube and conducting polymer composites for supercapacitors," Progress in Natural Science, vol. 18, no. 7, pp. 777-788, 2008.

[5] E. Frackowiak, V. Khomenko, K. Jurewicz, K. Lota, and F. Beguin, "Supercapacitors based on conducting polymers/nanotubes composites," Journal of Power Sources, vol. 153, no. 2, pp. 413-418, 2006.

[6] B. H. Kim, D. H. Park, J. Joo, S. G. Yu, and S. H. Lee, "Synthesis, characteristics, and field emission of doped and de-doped polypyrrole, polyaniline, poly(3,4-ethylenedioxythiophene) nanotubes and nanowires," Synthetic Metals, vol. 150, no. 3, pp. 279-284, 2005.

[7] T. Ahuja, I. A. Mir, D. Kumar, and Rajesh, "Biomolecular immobilization on conducting polymers for biosensing applications," Biomaterials, vol. 28, no. 5, pp. 791-805, 2007.

[8] R. Gangopadhyay and A. De, "Conducting polymer composites: novel materials for gas sensing," Sensors and Actuators B: Chemical, vol. 77, no. 1-2, pp. 326-329, 2001.

[9] A. D. Bendrea, L. Cianga, and I. Cianga, "Review paper: progress in the field of conducting polymers for tissue engineering applications," Journal of Biomaterials Applications, vol. 26, no. 1, pp. 3-84, 2011

[10] T. K. Das and S. Prusty, "Review on conducting polymers and their applications," Polymer - Plastics Technology and Engineering, vol. 51, no. 14, pp. 1487-1500, 2012.

[11] H. D. Tran, D. Li, and R. B. Kaner, "One-dimensional conducting polymer nanostructures: bulk synthesis and applications," Advanced Materials, vol. 21, no. 14-15, pp. 1487-1499, 2009.

[12] J. Yang, Y. Liu, S. Liu, L. Li, C. Zhang, and T. Liu, "Conducting polymer composites: material synthesis and applications in electrochemical capacitive energy storage," Materials Chemistry Frontiers, vol. 1, no. 2, pp. 251-268, 2017.

[13] J. H. Johnston, F. M. Kelly, J. Moraes, T. Borrmann, and D. Flynn, "Conducting polymer composites with cellulose and protein fibres," Current Applied Physics, vol. 6, no. 3, pp. 587-590, 2006.

[14] X. Lu, W. Zhang, C. Wang, T. C. Wen, and Y. Wei, "Onedimensional conducting polymer nanocomposites: synthesis, properties and applications," Progress in Polymer Science, vol. 36, no. 5, pp. 671-712, 2011.

[15] S. Kulandaivalu, Z. Zainal, and Y. Sulaiman, "Influence of monomer concentration on the morphologies and electrochemical properties of PEDOT, PANI, and PPy prepared from aqueous solution," International Journal of Polymer Science, vol. 2016, Article ID 8518293, 12 pages, 2016.

[16] W. Y. Hong, S. H. Jeon, E. S. Lee, and Y. Cho, “An integrated multifunctional platform based on biotin-doped conducting polymer nanowires for cell capture, release, and electrochemical sensing," Biomaterials, vol. 35, no. 36, pp. 9573-9580, 2014.

[17] Y. Liu, N. Lu, S. Poyraz et al., "One-pot formation of multifunctional Pt-conducting polymer intercalated nanostructures," Nanoscale, vol. 5, no. 9, pp. 3872-3879, 2013.

[18] B. Guo and P. X. Ma, "Conducting polymers for tissue engineering," Biomacromolecules, vol. 19, no. 6, pp. 1764-1782, 2018.

[19] A. Vaitkuviene, V. Kaseta, J. Voronovic et al., "Evaluation of cytotoxicity of polypyrrole nanoparticles synthesized by oxidative polymerization," Journal of Hazardous Materials, vol. 250-251, pp. 167-174, 2013.

[20] A. Vaitkuviene, V. Ratautaite, L. Mikoliunaite et al., "Some biocompatibility aspects of conducting polymer polypyrrole evaluated with bone marrow-derived stem cells," Colloids and Surfaces A: Physicochemical and Engineering Aspects, vol. 442, pp. 152-156, 2014.

[21] X. Wang, X. Gu, C. Yuan et al., "Evaluation of biocompatibility of polypyrrole in vitro and in vivo," Journal of Biomedical Materials Research Part A, vol. 68, no. 3, pp. 411-422, 2004.

[22] P. M. George, A. W. Lyckman, D. A. LaVan et al., "Fabrication and biocompatibility of polypyrrole implants suitable for neural prosthetics," Biomaterials, vol. 26, no. 17, pp. 3511-3519, 2005.

[23] A. Ramanaviciene, A. Kausaite, S. Tautkus, and A. Ramanavicius, "Biocompatibility of polypyrrole particles: an in-vivo study in mice," The Journal of Pharmacy and Pharmacology, vol. 59, no. 2, pp. 311-315, 2007.

[24] S. E. Moulton, M. J. Higgins, R. M. I. Kapsa, and G. G. Wallace, "Organic bionics: a new dimension in neural communications," Advanced Functional Materials, vol. 22, no. 10, pp. 2003-2014, 2012.

[25] R. Jalili, A. Kanneganti, M. I. Romero-Ortega, and G. G. Wallace, "Implantable electrodes," Current Opinion in Electrochemistry, vol. 3, no. 1, pp. 68-74, 2017.

[26] J. W. Lee, F. Serna, J. Nickels, and C. E. Schmidt, "Carboxylic acid-functionalized conductive polypyrrole as a bioactive platform for cell adhesion," Biomacromolecules, vol. 7, no. 6, pp. 1692-1695, 2006.

[27] H. Xu, J. M. Holzwarth, Y. Yan et al., "Conductive PPY/PDLLA conduit for peripheral nerve regeneration," Biomaterials, vol. 35, no. 1, pp. 225-235, 2014.

[28] Y. A. Ismail, S. R. Shin, K. M. Shin et al., "Electrochemical actuation in chitosan/polyaniline microfibers for artificial muscles fabricated using an in situ polymerization," Sensors and Actuators B: Chemical, vol. 129, no. 2, pp. 834-840, 2008. 
[29] H. S. Kim, H. L. Hobbs, L. Wang, M. J. Rutten, and C. C. Wamser, "Biocompatible composites of polyaniline nanofibers and collagen," Synthetic Metals, vol. 159, no. 13, pp. 1313-1318, 2009.

[30] B. Guo, A. Finne-Wistrand, and A. C. Albertsson, "Facile synthesis of degradable and electrically conductive polysaccharide hydrogels," Biomacromolecules, vol. 12, no. 7, pp. 2601-2609, 2011.

[31] R. A. Green, N. H. Lovell, and L. A. Poole-Warren, "Cell attachment functionality of bioactive conducting polymers for neural interfaces," Biomaterials, vol. 30, no. 22, pp. 3637-3644, 2009.

[32] G. Kaur, R. Adhikari, P. Cass, M. Bown, and P. Gunatillake, "Electrically conductive polymers and composites for biomedical applications," RSC Advances, vol. 5, no. 47, pp. 37553-37567, 2015.

[33] D. Svirskis, J. Travas-Sejdic, A. Rodgers, and S. Garg, "Electrochemically controlled drug delivery based on intrinsically conducting polymers," Journal of Controlled Release, vol. 146, no. 1, pp. 6-15, 2010.

[34] M. Gerard, A. Chaubey, and B. D. Malhotra, "Application of conducting polymers to biosensors," Biosensors \& Bioelectronics, vol. 17, no. 5, pp. 345-359, 2002.

[35] Rajesh, T. Ahuja, and D. Kumar, "Recent progress in the development of nano-structured conducting polymers/nanocomposites for sensor applications," Sensors and Actuators B: Chemical, vol. 136, no. 1, pp. 275-286, 2009.

[36] R. Langer, "Drug delivery and targeting," Nature, vol. 392, no. 6679, pp. 5-10, 1998.

[37] T. M. Allen and P. R. Cullis, "Drug delivery systems: entering the mainstream," Science, vol. 303, no. 5665, pp. 1818-1822, 2004.

[38] J. Folkman and D. M. Long, "The use of silicone rubber as a carrier for prolonged drug therapy," The Journal of Surgical Research, vol. 4, no. 3, pp. 139-142, 1964.

[39] K. S. Soppimath, T. M. Aminabhavi, A. R. Kulkarni, and W. E. Rudzinski, "Biodegradable polymeric nanoparticles as drug delivery devices," Journal of Controlled Release, vol. 70, no. 1-2, pp. 1-20, 2001.

[40] B. Zinger and L. L. Miller, "Timed release of chemicals from polypyrrole films," Journal of the American Chemical Society, vol. 106, no. 22, pp. 6861-6863, 1984.

[41] R. Wadhwa, C. F. Lagenaur, and X. T. Cui, "Electrochemically controlled release of dexamethasone from conducting polymer polypyrrole coated electrode," Journal of Controlled Release, vol. 110, no. 3, pp. 531-541, 2006.

[42] C. You, H. Wu, M. Wang et al., "Near-infrared light and $\mathrm{pH}$ dual-responsive targeted drug carrier based on corecrosslinked polyaniline nanoparticles for intracellular delivery of cisplatin," Chemistry - A European Journal, vol. 23, no. 22, pp. 5352-5360, 2017.

[43] M. R. Abidian, D. H. Kim, and D. C. Martin, "Conductingpolymer nanotubes for controlled drug release," Advanced Materials, vol. 18, no. 4, pp. 405-409, 2006.

[44] D. D. Ateh, H. A. Navsaria, and P. Vadgama, "Polypyrrolebased conducting polymers and interactions with biological tissues," Journal of The Royal Society Interface, vol. 3, no. 11, pp. 741-752, 2006.

[45] P. M. George, D. A. LaVan, J. A. Burdick, C. Y. Chen, E. Liang, and R. Langer, "Electrically controlled drug delivery from biotin-doped conductive polypyrrole," Advanced Materials, vol. 18, no. 5, pp. 577-581, 2006.

[46] K. M. Woeppel, X. S. Zheng, Z. M. Schulte, N. L. Rosi, and X. T. Cui, "Nanoparticle doped PEDOT for enhanced electrode coatings and drug delivery," Advanced Healthcare Materials, vol. 8, no. 21, article 1900622, 2019.

[47] D. H. Kim, S. M. Richardson-Burns, J. L. Hendricks, C. Sequera, and D. C. Martin, "Effect of immobilized nerve growth factor on conductive polymers: electrical properties and cellular response," Advanced Functional Materials, vol. 17, no. 1, pp. 79-86, 2007.

[48] A. J. Evans, B. C. Thompson, G. G. Wallace et al., "Promoting neurite outgrowth from spiral ganglion neuron explants using polypyrrole/BDNF-coated electrodes," Journal of Biomedical Materials Research Part A, vol. 91A, no. 1, pp. 241250, 2009.

[49] Y. Qiu and K. Park, "Environment-sensitive hydrogels for drug delivery," Advanced Drug Delivery Reviews, vol. 53, no. 3, pp. 321-339, 2001.

[50] S. Kalia, Polymeric hydrogels as smart biomaterials, Springer International Publishing, Switzerland, 2016.

[51] L. M. Low, S. Seetharaman, K. Q. He, and M. J. Madou, "Microactuators toward microvalves for responsive controlled drug delivery," Sensors and Actuators B: Chemical, vol. 67, no. 1-2, pp. 149-160, 2000.

[52] L. M. Lira and S. I. Córdoba de Torresi, "Conducting polymer-hydrogel composites for electrochemical release devices: synthesis and characterization of semiinterpenetrating polyaniline- polyacrylamide networks," Electrochemistry Communications, vol. 7, no. 7, pp. 717723,2005

[53] D. H. Kim, M. Abidian, and D. C. Martin, "Conducting polymers grown in hydrogel scaffolds coated on neural prosthetic devices," Journal of Biomedical Materials Research Part A, vol. 71, no. 4, pp. 577-585, 2004.

[54] J. Ge, E. Neofytou, T. J. Cahill II, R. E. Beygui, and R. N. Zare, "Drug release from electric-field-responsive nanoparticles," ACS Nano, vol. 6, no. 1, pp. 227-233, 2012.

[55] J. Qu, X. Zhao, P. X. Ma, and B. Guo, "Injectable antibacterial conductive hydrogels with dual response to an electric field and $\mathrm{pH}$ for localized "smart" drug release," Acta Biomaterialia, vol. 72, pp. 55-69, 2018.

[56] B. Guo, L. Glavas, and A. C. Albertsson, "Biodegradable and electrically conducting polymers for biomedical applications," Progress in Polymer Science, vol. 38, no. 9, pp. 1263 1286, 2013.

[57] M. A. Mahmoud, P. Szymanski, and M. A. El-Sayed, "Different methods of increasing the mechanical strength of gold nanocages," Journal of Physical Chemistry Letters, vol. 3, no. 23, pp. 3527-3531, 2012.

[58] M. A. Green, L. E. Bilston, and R. Sinkus, "In vivo brain viscoelastic properties measured by magnetic resonance elastography," NMR in Biomedicine, vol. 21, no. 7, pp. 755-764, 2008.

[59] T. Saxena, J. L. Gilbert, and J. M. Hasenwinkel, "A versatile mesoindentation system to evaluate the micromechanical properties of soft, hydrated substrates on a cellular scale," Journal of Biomedical Materials Research Part A, vol. 90A, no. 4, pp. 1206-1217, 2009.

[60] A. Y. Mekaj, A. A. Morina, S. Lajqi, S. Manxhuka-Kerliu, F. M. Kelmendi, and S. B. Duci, "Biomechanical properties 
of the sciatic nerve following repair: effects of topical application of hyaluronic acid or tacrolimus," International Journal of Clinical and Experimental Medicine, vol. 8, no. 11, pp. 20218-20226, 2015.

[61] G. H. Borschel, K. F. Kia, W. M. Kuzon Jr., and R. G. Dennis, "Mechanical properties of acellular peripheral nerve," The Journal of Surgical Research, vol. 114, no. 2, pp. 133-139, 2003.

[62] M. Ganji, A. C. Paulk, J. C. Yang et al., "Selective formation of porous Pt nanorods for highly electrochemically efficient neural electrode interfaces," Nano Letters, vol. 19, no. 9, pp. 6244-6254, 2019.

[63] D. Duc, P. R. Stoddart, S. L. McArthur et al., "Fabrication of a biocompatible liquid crystal graphene oxide-gold nanorods electro- and photoactive interface for cell stimulation," Advanced Healthcare Materials, vol. 8, no. 9, article $1801321,2019$.

[64] C. Chen, S. Ruan, X. Bai, C. Lin, C. Xie, and I. S. Lee, "Patterned iridium oxide film as neural electrode interface: biocompatibility and improved neurite outgrowth with electrical stimulation," Materials Science and Engineering: C, vol. 103, article 109865, 2019.

[65] N. Torres-Martinez, C. Cretallaz, D. Ratel et al., "Evaluation of chronically implanted subdural boron doped diamond/CNT recording electrodes in miniature swine brain," Bioelectrochemistry, vol. 129, pp. 79-89, 2019.

[66] G. A. Snook, P. Kao, and A. S. Best, "Conducting-polymerbased supercapacitor devices and electrodes," Journal of Power Sources, vol. 196, no. 1, pp. 1-12, 2011.

[67] A. R. Harris, S. Morgan, J. Chen, R. M. I. Kapsa, G. G. Wallace, and A. G. Paolini, "Conducting polymer coated neural recording electrodes," Journal of Neural Engineering, vol. 10, no. 1, article 016004, 2013.

[68] P. Zarrintaj, Z. Ahmadi, H. Vahabi, F. Ducos, M. R. Saeb, and M. Mozafari, "Polyaniline in retrospect and prospect," Materials Today: Proceedings, vol. 5, no. 7, pp. 15852-15860, 2018.

[69] S. Lee, T. Eom, M. K. Kim, S. G. Yang, and B. S. Shim, "Durable soft neural micro-electrode coating by an electrochemical synthesis of PEDOT:PSS / graphene oxide composites," Electrochimica Acta, vol. 313, pp. 79-90, 2019.

[70] H. Zhou, X. Cheng, L. Rao, T. Li, and Y. Y. Duan, "Poly(3,4ethylenedioxythiophene)/multiwall carbon nanotube composite coatings for improving the stability of microelectrodes in neural prostheses applications," Acta Biomaterialia, vol. 9, no. 5, pp. 6439-6449, 2013.

[71] C. Bodart, N. Rossetti, J.'. E. Hagler et al., "Electropolymerized Poly(3,4-ethylenedioxythiophene) (PEDOT) coatings for implantable deep-brain-stimulating microelectrodes," ACS Applied Materials \& Interfaces, vol. 11, no. 19, pp. 17226-17233, 2019.

[72] X. Cui, V. A. Lee, Y. Raphael et al., "Surface modification of neural recording electrodes with conducting polymer/biomolecule blends," Journal of Biomedical Materials Research, vol. 56, no. 2, pp. 261-272, 2001.

[73] A. M. Cobo, C. E. Larson, K. Scholten et al., "Parylene-based cuff electrode with integrated microfluidics for peripheral nerve recording, stimulation, and drug delivery," Journal of Microelectromechanical Systems, vol. 28, no. 1, pp. 36-49, 2019.

[74] S. Biswas, D. Sikdar, D. Das, M. Mahadevappa, and S. Das, "PDMS based multielectrode arrays for superior in-vitro ret- inal stimulation and recording," Biomedical Microdevices, vol. 19 , no. 4 , p. $75,2017$.

[75] M. S. Nahvi, F. A. Boroumand, M. H. Maghami, A. M. Sodagar, A. Shojaei, and J. Mirnajafi-Zadeh, "Design, fabrication, and test of flexible thin-film microelectrode arrays for neural interfaces," in 2017 IEEE 30th Canadian Conference on Electrical and Computer Engineering, Windsor, Canada, April 2017.

[76] W. F. Gillis, C. A. Lissandrello, J. Shen et al., "Carbon fiber on polyimide ultra-microelectrodes," Journal of Neural Engineering, vol. 15, no. 1, article 016010, 2018.

[77] Z. Shi, F. Zheng, Z. Zhou et al., "Silk-enabled conformal multifunctional bioelectronics for investigation of spatiotemporal epileptiform activities and multimodal neural encoding/decoding," Advancement of Science, vol. 6, no. 9, article 1801617, 2019.

[78] D. N. Heo, H. J. Kim, Y. J. Lee et al., "Flexible and highly biocompatible nanofiber-based electrodes for neural surface interfacing," ACS Nano, vol. 11, no. 3, pp. 2961-2971, 2017.

[79] C. Wang, W. Zheng, Z. Yue, C. O. Too, and G. G. Wallace, "Buckled, stretchable polypyrrole electrodes for battery applications," Advanced Materials, vol. 23, no. 31, pp. 3580-3584, 2011.

[80] T. S. Hansen, K. West, O. Hassager, and N. B. Larsen, "Highly stretchable and conductive polymer material made from poly(3,4-ethylenedioxythiophene) and polyurethane elastomers," Advanced Functional Materials, vol. 17, no. 16, pp. 3069-3073, 2007.

[81] R. Samba, T. Herrmann, and G. Zeck, "PEDOT-CNT coated electrodes stimulate retinal neurons at low voltage amplitudes and low charge densities," Journal of Neural Engineering, vol. 12, no. 1, article 016014, 2015.

[82] N. C. Foulds and C. R. Lowe, "Enzyme entrapment in electrically conducting polymers. Immobilisation of glucose oxidase in polypyrrole and its application in amperometric glucose sensors," Journal of the Chemical Society, Faraday Transactions 1: Physical Chemistry in Condensed Phases, vol. 82, no. 4, pp. 1259-1264, 1986.

[83] R. Janmanee, S. Chuekachang, S. Sriwichai, A. Baba, and S. Phanichphant, "Functional conducting polymers in the application of SPR biosensors," Journal of Nanotechnology, vol. 2012, Article ID 620309, 7 pages, 2012.

[84] R. A. Green, N. H. Lovell, and L. A. Poole-Warren, "Impact of co-incorporating laminin peptide dopants and neurotrophic growth factors on conducting polymer properties," Acta Biomaterialia, vol. 6, no. 1, pp. 63-71, 2010.

[85] C. M. Li, W. Chen, X. Yang et al., "Impedance labelless detection-based polypyrrole protein biosensor," Frontiers in Bioscience, vol. 10, no. 1-3, pp. 2518-2526, 2005.

[86] J. Park, S. Choi, A. H. Janardhan et al., "Electromechanical cardioplasty using a wrapped elasto-conductive epicardial mesh," Science Translational Medicine, vol. 8, no. 344, p. 344ra86, 2016.

[87] J. W. Costerton, L. Montanaro, and C. R. Arciola, "Biofilm in implant infections: its production and regulation," The International Journal of Artificial Organs, vol. 28, no. 11, pp. 1062 1068, 2005.

[88] D. H. Kim, Y. S. Kim, J. Amsden et al., "Silicon electronics on silk as a path to bioresorbable, implantable devices," Applied Physics Letters, vol. 95, no. 13, p. 133701, 2009. 
[89] H. K. Makadia and S. J. Siegel, "Poly lactic-co-glycolic acid (PLGA) as biodegradable controlled drug delivery carrier," Polymers, vol. 3, no. 3, pp. 1377-1397, 2011.

[90] H. Tao, S. W. Hwang, B. Marelli et al., "Silk-based resorbable electronic devices for remotely controlled therapy and in vivo infection abatement," Proceedings of the National Academy of Sciences of the United States of America, vol. 111, no. 49, pp. 17385-17389, 2014.

[91] R. Feiner and T. Dvir, "Tissue-electronics interfaces: from implantable devices to engineered tissues," Nature Reviews Materials, vol. 3, no. 1, article 317076, 2018.

[92] P. R. Troyk, "Injectable electronic identification, monitoring, and stimulation systems," Annual Review of Biomedical Engineering, vol. 1, no. 1, pp. 177-209, 1999.

[93] G. Hong, T. M. Fu, M. Qiao et al., "A method for singleneuron chronic recording from the retina in awake mice," Science, vol. 360, no. 6396, pp. 1447-1451, 2018.

[94] T. Zhou, G. Hong, T. M. Fu et al., "Syringe-injectable mesh electronics integrate seamlessly with minimal chronic immune response in the brain," Proceedings of the National Academy of Sciences of the United States of America, vol. 114, no. 23, pp. 5894-5899, 2017.

[95] J. Liu, T. M. Fu, Z. Cheng et al., "Syringe-injectable electronics," Nature Nanotechnology, vol. 10, no. 7, pp. 629-636, 2015.

[96] G. Hong, T. M. Fu, T. Zhou, T. G. Schuhmann, J. Huang, and C. M. Lieber, "Syringe injectable electronics: precise targeted delivery with quantitative input/output connectivity," Nano Letters, vol. 15, no. 10, pp. 6979-6984, 2015.

[97] T. M. Fu, G. Hong, T. Zhou, T. G. Schuhmann, R. D. Viveros, and C. M. Lieber, "Stable long-term chronic brain mapping at the single-neuron level," Nature Methods, vol. 13, no. 10, pp. 875-882, 2016.

[98] T. G. Schuhmann Jr., J. Yao, G. Hong, T.-M. Fu, and C. M. Lieber, "Syringe-injectable electronics with a plug-and-play input/output interface," Nano Letters, vol. 17, no. 9, pp. 5836-5842, 2017.

[99] T. M. Fu, G. Hong, R. D. Viveros, T. Zhou, and C. M. Lieber, "Highly scalable multichannel mesh electronics for stable chronic brain electrophysiology," Proceedings of the National Academy of Sciences of the United States of America, vol. 114, no. 47, pp. E10046-E10055, 2017.

[100] R. D. Viveros, T. Zhou, G. Hong, T. M. Fu, H. Y. G. Lin, and C. M. Lieber, "Advanced one- and two-dimensional mesh designs for injectable electronics," Nano Letters, vol. 19, no. 6, pp. 4180-4187, 2019.

[101] K. V. Nemani, K. L. Moodie, J. B. Brennick, A. Su, and B. Gimi, "In vitro and in vivo evaluation of SU-8 biocompatibility," Materials Science and Engineering: C, vol. 33, no. 7, pp. 4453-4459, 2013.

[102] X. Dai, G. Hong, T. Gao, and C. M. Lieber, "Mesh nanoelectronics: seamless integration of electronics with tissues," Accounts of Chemical Research, vol. 51, no. 2, pp. 309-318, 2018.

[103] B. Tian, J. Liu, T. Dvir et al., "Macroporous nanowire nanoelectronic scaffolds for synthetic tissues," Nature Materials, vol. 11, no. 11, pp. 986-994, 2012.

[104] X. Yang, T. Zhou, T. J. Zwang et al., "Bioinspired neuron-like electronics," Nature Materials, vol. 18, no. 5, pp. 510-517, 2019.
[105] L. Luan, X. Wei, Z. Zhao et al., "Ultraflexible nanoelectronic probes form reliable, glial scar-free neural integration," Science Advances, vol. 3, no. 2, article e1601966, 2017.

[106] A. P. Alivisatos, A. M. Andrews, E. S. Boyden et al., "Nanotools for neuroscience and brain activity mapping," ACS Nano, vol. 7, no. 3, pp. 1850-1866, 2013.

[107] J. Rivnay, H. Wang, L. Fenno, K. Deisseroth, and G. G. Malliaras, "Next-generation probes, particles, and proteins for neural interfacing," Science Advances, vol. 3, no. 6, article e1601649, 2017.

[108] H. Tong, K. Lou, and W. Wang, "Near-infrared fluorescent probes for imaging of amyloid plaques in Alzheimer's disease," Acta Pharmaceutica Sinica B, vol. 5, no. 1, pp. 25-33, 2015.

[109] G. Kook, S. W. Lee, H. C. Lee, I.-J. Cho, and H. J. Lee, "Neural probes for chronic applications," Micromachines, vol. 7, no. 10, p. 179, 2016.

[110] H.-Y. Lai, L. D. Liao, C. T. Lin et al., "Design, simulation and experimental validation of a novel flexible neural probe for deep brain stimulation and multichannel recording," Journal of Neural Engineering, vol. 9, no. 3, article 036001, 2012.

[111] T. Kim, J. G. McCall, Y. H. Jung et al., "Injectable, cellularscale optoelectronics with applications for wireless optogenetics," Science, vol. 340, no. 6129, pp. 211-216, 2013. 